\title{
Design of Internal Wire-Based Impedance Matching of Helical Antennas Using an Equivalent Thin-Wire Model
}

\author{
Slobodan V. Savić, ${ }^{1}$ Milan M. Ilić, ${ }^{1,2}$ and Antonije R. Djordjević ${ }^{1,3}$ \\ ${ }^{1}$ School of Electrical Engineering, University of Belgrade, Bulevar kralja Aleksandra 73, 11120 Belgrade, Serbia \\ ${ }^{2}$ Department of Electrical and Computer Engineering, Colorado State University, Fort Collins, CO 80523-1373, USA \\ ${ }^{3}$ Serbian Academy of Sciences and Arts, Knez Mihailova 35, 11000 Belgrade, Serbia
}

Correspondence should be addressed to Milan M. Ilić; milanilic@etf.rs

Received 9 August 2017; Accepted 23 November 2017; Published 17 December 2017

Academic Editor: Miguel Ferrando Bataller

Copyright (c) 2017 Slobodan V. Savić et al. This is an open access article distributed under the Creative Commons Attribution License, which permits unrestricted use, distribution, and reproduction in any medium, provided the original work is properly cited.

\begin{abstract}
We propose a novel design of internal impedance matching networks for axial-mode helical antennas. This network comprises a single wire attached to the helix. One of the main challenges when designing an internal matching network is its strong electromagnetic coupling with the antenna. The matching network must hence be designed in the presence of the antenna, which slows down the design process. To overcome this problem, we formulate an equivalent thin-wire model of the complete helix, including the matching wire (matching network) and the dielectric support. This computationally low-demanding model can be analyzed extremely rapidly, yielding accurate results, which are in excellent agreement with alternative numerical solutions and measurements.
\end{abstract}

\section{Introduction}

Axial-mode helical antennas have been known for a long time [1-8]. Inherently, they are poorly matched to the standard $50 \Omega$ transmission lines. However, they can be matched utilizing classical external matching networks (located beyond the antenna port) or utilizing metallic and dielectric features (internal) incorporated into the antenna structure [7, 9-12].

The input impedance of axial-mode helical antennas is close to $150 \Omega[2,13]$, and a well-designed internal matching can have good performance with minimal complexity and price overhead [12]. In [9], it was demonstrated that the input impedance of axial-mode helical antennas strongly depends on the shape of the beginning of its conductor. A helical antenna internally matched by an impedance transformer similar to a transmission line structure, comprising the helix reflector and a metallic plate horizontally attached to the beginning of the helical conductor, was presented in [10]. This notable work represents a proof of concept, with somewhat incomplete consideration of practical aspects.
Namely, a helical conductor is usually wound around a cylindrical mount, and when a helix is circumferentially fed, the matching from [10] is not always practical because the metallic plate and the mount intersect. Following a similar idea, the antenna in [13] is internally matched by adjusting the spacing between the beginning of the helical conductor and the reflector. However, the profile of the internal matching presented in [13] requires a relatively complex calculation [14] and employs a nonuniform helix.

An alternative solution, an impedance transformer similar to a quarter-wave transformer, comprising the helix reflector and a metallic plate vertically attached to the beginning of the helical conductor, was presented in $[7,11,12]$. The characteristic impedance of this transformer should be smaller than the characteristic impedance of the transmission line formed by the beginning of the helical conductor and the reflector, as it transforms the helix input impedance (close to $150 \Omega$ ) to $50 \Omega$. Thus, the added metallic plate should decrease the characteristic impedance of the transmission line formed by the helical conductor and the reflector; namely, its per-unit-length (PUL) capacitance should 


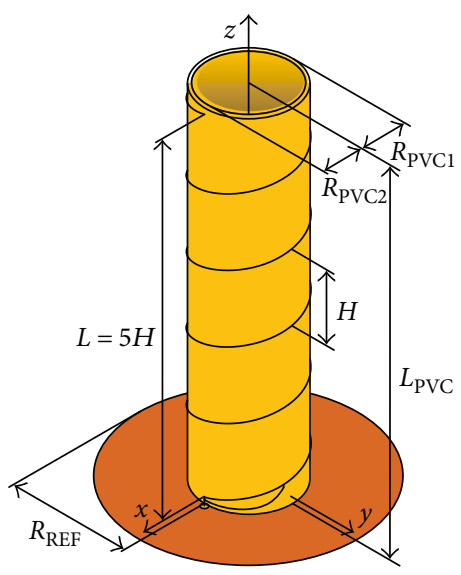

(a)

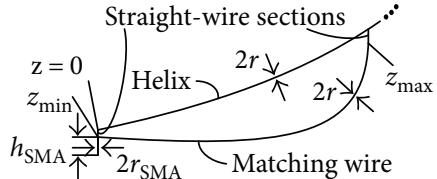

(b)

Figure 1: (a) A helical antenna with a matching wire. (b) A sketch of the matching wire, whose geometry is governed by (1).

be increased and hence the metallic plate should preferably be attached to the bottom of the helical conductor $[7,12]$ (as close as needed to the helix reflector), as opposed to the solution presented in [11].

Following these ideas, originated from [10,13], with the modifications first proposed in [7] and verified in [12], we here propose an internal impedance matching network for a helical antenna which comprises a single wire. We recognize that one of the main challenges when designing an internal matching is its strong electromagnetic (EM) coupling with the antenna. The matching network must therefore be designed in the presence of the antenna by means of either relatively lengthy and expensive prototype tuning [11] or complex full-wave numerical analysis [12]. In addition, we formulate here a wire-based equivalent (simplified) model of the internally matched helix. This computationally lowdemanding model enables fast and accurate design of internally matched helical antennas with dielectric supports. The model is based on the equivalent model of wires mounted in the vicinity of dielectric and/or magnetic objects [15] and yields very accurate results.

\section{Internally Matched Helix}

We consider a helical antenna made of a copper wire with a radius $r=0.525 \mathrm{~mm}$, wound around a hollow PVC tube with an outer radius $R_{\mathrm{PVC} 1}=80 \mathrm{~mm}$ and an inner radius $R_{\mathrm{PVC} 2}=$ $76 \mathrm{~mm}$, as shown in Figure 1(a). Copper conductivity is $\sigma_{\mathrm{Cu}}=58 \mathrm{MS} / \mathrm{m}$, and PVC dielectric parameters are $\varepsilon_{r}=2.8$ and $\tan \delta=0.0049$. The helix starts at $z=0$ and has $N=5$ uniform turns with pitch length $H=120 \mathrm{~mm}$ (the helix total length is $L=N H=600 \mathrm{~mm}$ ). On both ends of the helix, the PVC tube is extended by $7.5 \mathrm{~mm}$ (the total length of the PVC tube is $L_{\mathrm{PVC}}=615 \mathrm{~mm}$ ). A circular copper reflector with radius $R_{\mathrm{REF}}=200 \mathrm{~mm}$ is placed at the bottom end of the PVC tube (at $z=-7.5 \mathrm{~mm}$ ). The helix is circumferentially fed via an SMA connector mounted at the helix periphery through a hole in the reflector.

Next, we propose a matching network made of a single wire connected to the beginning of the helical conductor, as shown in Figure 1. When the matching network and the helix are made solely of wires wound around a PVC tube with a reflector, starting from the theory presented in [15], it is relatively simple to formulate an efficient and accurate equivalent wire-based numerical model of the internally matched helix. This equivalent model, formulated in Section 3, enables rapid computational simulation even using low-end computers. It thus provides means for extremely quick, accurate, and easy computer-aided design of a matched helix and overcomes the need for the relatively lengthy prototype tuning or full-wave computational simulations of complete numerical models.

The matching wire is made of the same copper wire as that in the helix (this will further simplify the formulation of the equivalent model). The geometry of the matching wire is parameterized by the following equations in Cartesian coordinates, as in [12]:

$$
\begin{aligned}
& x=\left(R_{\mathrm{PVC} 1}+r\right) \cos \left(\frac{\pi}{2} t\right), \\
& y=\left(R_{\mathrm{PVC} 1}+r\right) \sin \left(\frac{\pi}{2} t\right), \\
& z=z_{\min }+\frac{z_{\max }-z_{\min }}{10^{p_{p}}-1}\left(10^{p_{p}\left(\frac{t}{t_{\max }}\right)}-1\right), \quad 0 \leq t \leq t_{\max } .
\end{aligned}
$$

The overall geometrical profile of the matching wire is determined by the parameter $p_{p}$, whereas $t_{\max }$ determines the length of the matching wire (for $t_{\max }=1$, the matching wire extends to one quarter of the first helical turn). The ends of the matching wire, having coordinates $z=z_{\min }$ and $z=z_{\max }$, are connected to the helix by vertical straight-wire sections, as shown in Figure 1(b). The SMA inner conductor is attached to the matching wire. Its radius and height are $r_{\mathrm{SMA}}=0.635$ $\mathrm{mm}$ and $h_{\mathrm{SMA}}=5.25 \mathrm{~mm}$, respectively.

The parametrization (1) was introduced in [12] to describe the bottom edge of a matching plate. It defines a curve utilizing a small number of parameters $\left(z_{\min }, z_{\max }\right.$, $t_{\max }$, and $\left.p_{p}\right)$. The curve is smoothly shaped, with no abrupt changes in its geometry. Of course, other parametrization schemes can be used.

As it will be described in Section 4, once parameters from (1) have been determined via an optimization or tuning 


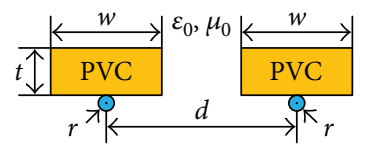

(a)

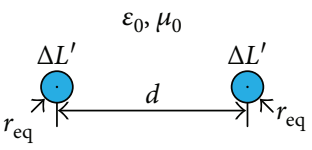

(b)
Figure 2: Cross sections of (a) the original and (b) the equivalent two-wire transmission lines.

process, the construction of the matching wire is simple and it does not contribute to the antenna complexity or price. Moreover, the matching wire can be easily tuned on the fabricated prototype, if such a demand would arise. This is not always the case with alternative matching designs.

\section{Equivalent Model}

When a helix is internally matched by a vertically profiled metallic plate $[7,11,12]$ wound around a PVC tube, an accurate model cannot be formulated easily. As opposed to this, when a helix is internally matched by a wire, an equivalent model is relatively easy to formulate, especially when the matching is made of the same wire as that in the helix. In that case, the helix and the matching wire, both wound around a PVC tube, can be equivalently replaced by metallic wires (with distributed loadings) situated in a free space [15]. In other words, rather than explicitly taking into account the presence of the PVC tube (utilizing the complete EM models), the PVC tube is considered implicitly, utilizing the equivalent wire model.

The complete models of the matched helix are typically tailored for simulations to be carried out by the most commonly used rigorous full-wave numerical techniques, that is, the finite element method (FEM) or the method of moments (MoM). In comparison with these models, the equivalent wire model is much more computationally efficient, especially when wires are simulated by the MoM $[16,17]$ utilizing the thin-wire approximation [18]. Moreover, the formulation of the highly accurate complete MoM model, which explicitly takes into account the PVC tube in the vicinity of a wire, is not a trivial task. This is an additional motivation for the formulation of the equivalent wire model, and it is described below.

Consider a short wire segment wound around a PVC tube. Since the curvature of this structure is relatively small, the wire can be locally considered straight, laying down on a flat PVC substrate. We expect that neglecting the curvature of this structure will not significantly affect the validity of equivalence and that we can introduce uniform transmission lines necessary for the derivation of the equivalence [15].

Contemplating structures suitable for the equivalence, we consider two-wire transmission lines (a) with a PVC substrate and (b) without a PVC substrate, as described in [15] and depicted in Figure 2. The line conductors of the original twowire transmission line lay on a $t=R_{\mathrm{PVC} 1}-R_{\mathrm{PVC} 2}=4 \mathrm{~mm}$ thick PVC substrate, as shown in Figure 2(a). The thickness of the PVC substrate is equal to the thickness of the wall of the PVC tube, and the original two-wire transmission line conductors are made of the same wire as that in the helix. Characteristics of this transmission line are mostly influenced by the
PVC in the proximity to the conductors. Hence, through numerical experiments, we set the width of the substrate to be $w=10 t=40 \mathrm{~mm}$. The distance between the conductors of the line is set to $d=10 w=400 \mathrm{~mm}$, as recommended in [15]. Employing the quasi-static analysis $[19,20]$, we evaluate the PUL capacitance of the line to be $C^{\prime}=4.28 \mathrm{pF} / \mathrm{m}$ and the inductance to be $L^{\prime}=2.75 \mu \mathrm{H} / \mathrm{m}$.

The cross section of the equivalent two-wire transmission line is shown in Figure 2(b). The equivalent transmission line has no PVC substrate, and its conductor radius $\left(r_{\text {eq }}\right)$ is evaluated so that the original and the equivalent transmission lines have the same PUL capacitance [15]. To equalize the PUL inductance of the original and the equivalent transmission lines [15], a series PUL inductance $\Delta L^{\prime}$ is added to each conductor of the equivalent transmission line in the numerical model.

By employing the quasi-static analysis $[19,20]$, the wire radii and the PUL inductance of the equivalent transmission line are evaluated to be $r_{\mathrm{eq}}=1.5 \mathrm{~mm}$ and $L_{\mathrm{eq}}^{\prime}=2.34 \mu \mathrm{H} / \mathrm{m}$, respectively, so the series PUL inductance necessary for the equivalence is $\Delta L^{\prime}=0.205 \mu \mathrm{H} / \mathrm{m}\left[\Delta L^{\prime}=\left(L^{\prime}-L_{\mathrm{eq}}^{\prime}\right) / 2\right]$. The parameters of the equivalent two-wire transmission line ( $r_{\text {eq }}$ and $\Delta L^{\prime}$ ) will be used when constructing the equivalent wire model of the internally matched helix. In the equivalent wire model of the matched helix, all wires (helix and matching), having the same radius $\left(r_{\mathrm{eq}}\right)$, are situated in a free space (with no PVC tube), with the series PUL inductance $\Delta L^{\prime}$ included as a distributed loading.

Adding the PUL inductance to a wire is a standard feature in modern full-wave EM simulators [16, 17]. It only alters the boundary conditions and practically does not increase the model complexity and simulation run time. We next evaluate the efficiency and accuracy of the proposed equivalent model of the internally matched helix by comparing our simulation results with full-wave simulations $[17,21]$ of the complete model and with measurements on a fabricated prototype.

\section{Results}

The equivalent (purely metallic, MoM) model of the helix with the matching wire is assembled in the software WIPL-D Pro [17]. The analysis is based on the thin-wire approximation [18]; it requires 2103 unknowns and lasts 4 seconds per frequency point, when run on a modest PC. The parameters of the matching wire in (1) are tuned so that the helix is best matched (its reflection coefficient with respect to $50 \Omega$ is minimal) in the vicinity of $600 \mathrm{MHz}$. The parameters $z_{\min }=-2.25 \mathrm{~mm}$ and $z_{\max }=24.41 \mathrm{~mm}$ are predefined, and the tuning yielded $t_{\max }=0.8804$ and $p_{p}=2.609$.

The complete full-wave FEM model (with the explicit presence of the PVC tube) of the matched helix is assembled in the software ANSYS HFSS [21]. It requires 420,929 unknowns and lasts 8 minutes and 14 seconds per frequency point (only the final adaptive pass is considered) on the same PC. The reflection coefficient obtained by utilizing the equivalent wire model and the complete full-wave FEM model is 


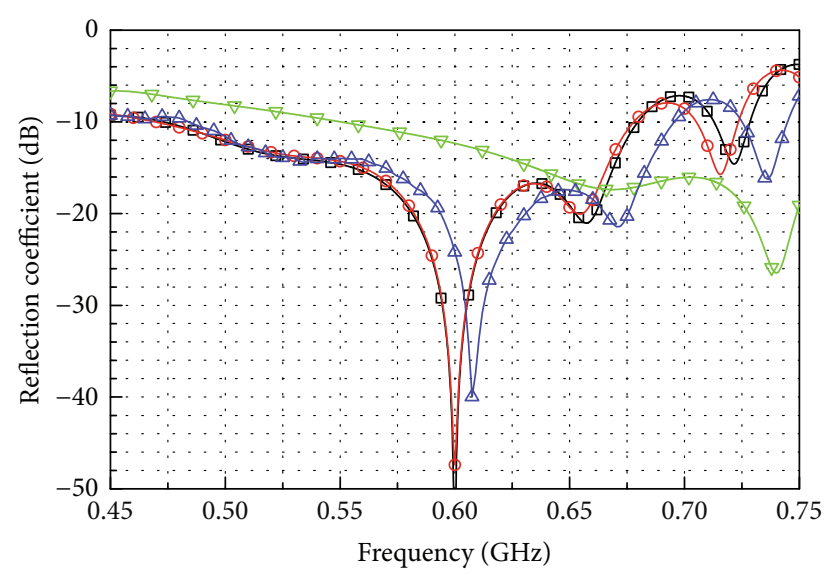

\begin{tabular}{rlr} 
& Model & \multicolumn{2}{c}{ Unknowns Time per freq. } \\
$\square-$ Equivalent wire (WIPL-D) & --2103 & $4 \mathrm{~s}$ \\
- - Complete (HFSS) & -420929 & $8 \mathrm{~min} 14 \mathrm{~s}$ \\
$\nabla$ Incomplete wire (WIPL-D) & $-\nabla 2103$ & $4 \mathrm{~s}$ \\
$\triangle$ & &
\end{tabular}

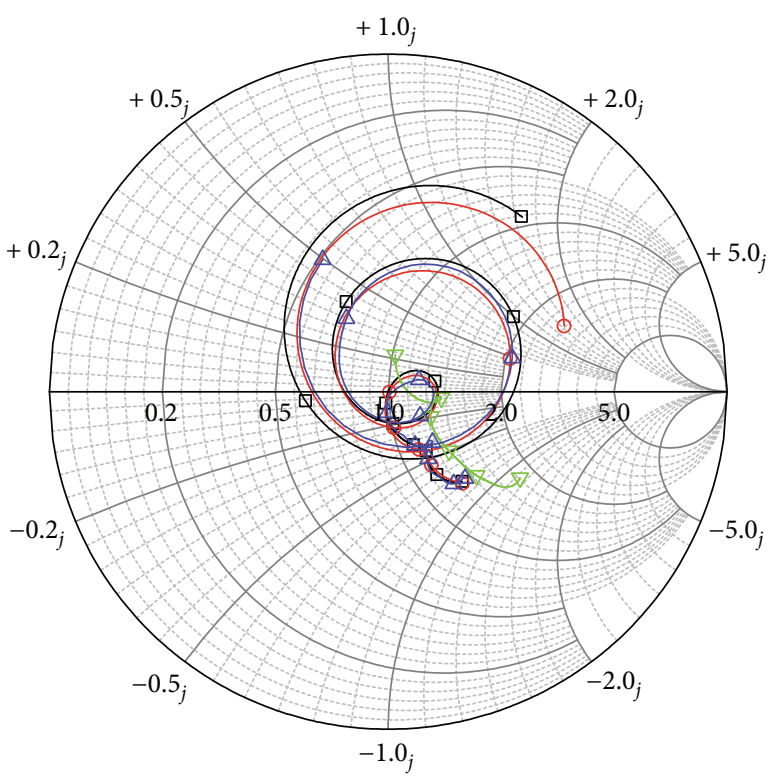

Model

$\square$ Equivalent wire (WIPL-D)

- - Complete (HFSS)

$\nabla$ Incomplete wire (WIPL-D)

$\triangle$ Prototype measurements

FIgURE 3: The reflection coefficient of the helical antenna with the matching wire: comparison of three simulation models and measurements.

shown in Figure 3. Note that WIPL-D Pro and ANSYS HFSS utilize different numerical techniques, MoM and FEM, respectively. Hence, this comparison provides a good crosscheck combination for unbiased evaluation of the accuracy of the obtained results. As it can be seen from Figure 3, these two sets of results are in an excellent agreement. In addition, note that when the complete full-wave MoM model (with the explicit presence of the PVC tube) is assembled in WIPL-D Pro (the results were not shown for brevity), the simulation requires 34,713 unknowns and lasts 31 minutes and 58

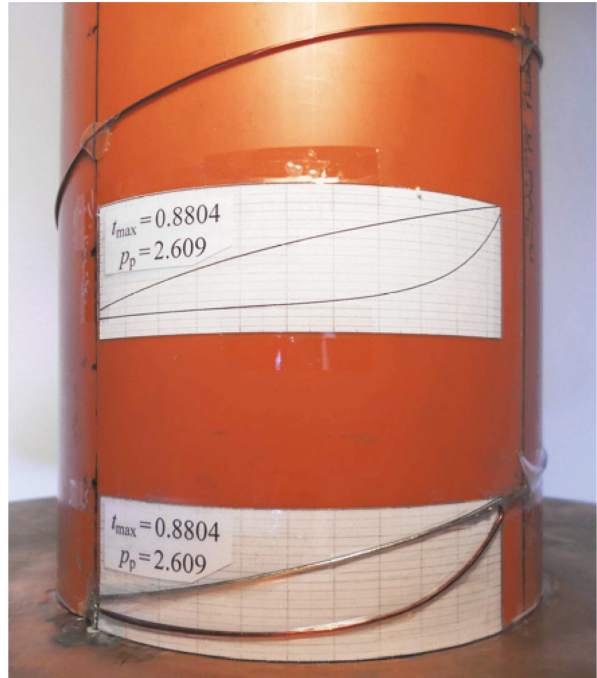

Figure 4: A photograph of the internally matched helix prototype. The stencil for the matching wire, preprinted on a piece of paper, is also shown.

seconds per frequency point on the same PC. We can conclude that, in this example, the equivalent wire model yields a reduction of the simulation time by more than 100 times when compared to the FEM model and by more than 400 times when compared to the MoM complete model.

Also presented in Figure 3 are the results obtained by measurements on the fabricated matched antenna, for which a detail presenting the first two turns is shown in Figure 4. Acknowledging prototype fabrication tolerances, we conclude that the measurements and numerical results are in a very good agreement.

Finally, we will examine the influence of the PVC tube on the helix reflection coefficient. By starting from the complete WIPL-D Pro model, we simply omit the PVC tube. Thus obtained "incomplete" wire results are also presented in Figure 3. We can conclude from Figure 3 that the results obtained utilizing the "incomplete" model significantly deviate from both the other numerical results and measurements. We thus confirm that, for the fast and accurate design of helical antennas with a dielectric tube, the tube cannot be simply omitted and the formulated equivalent wire model represents an excellent choice.

\section{Conclusions}

We have proposed a simple and inexpensive wire-based internal matching network for axial-mode helical antennas. We have also formulated an equivalent thin-wire-based EM model of internally matched helical antennas. The proposed model yields results which are in an excellent agreement with the complete full-wave FEM simulations. The accuracy of the proposed model has also been validated by measurements on a fabricated antenna. In the presented example, the proposed model reduces the simulation run time by more than 100 times and 400 times, compared to FEM and MoM fullwave analysis of complete models, respectively, while maintaining excellent accuracy. We remark that when employing 
the equivalence from [15], as done here, high-permittivity dielectrics may yield unacceptably large wire radii in the equivalent model. This can be bypassed by using the method from [22]. In that method, a wire coated with a dielectric layer is treated by considering currents and charges that are located on the wire surface with an additional layer of charges located at the outer surface of the coating. However, in the available commercial software, only the currents and charges located on the wire surface are involved in the thin-wire model. Hence, the application of the method from [22] requires writing a customized code.

\section{Conflicts of Interest}

The authors declare that there is no conflict of interest regarding the publication of this article.

\section{Acknowledgments}

This work was supported in part by the Ministry of Education, Science and Technological Development of Serbia under Grant TR32005.

\section{References}

[1] G. A. Thiele and W. L. Stutzman, Antenna Theory and Design, J. Wiley, New York, 1981.

[2] J. D. Kraus, Antennas, (Electrical Engineering), McGraw-Hill, New York, 2nd edition, 1988.

[3] J. D. Kraus, "Helical beam antennas," Electronics, vol. 20, pp. 109-111, 1947.

[4] J. D. Kraus and J. C. Williamson, "Characteristics of helical antennas radiating in the axial mode," Journal of Applied Physics, vol. 19, no. 1, pp. 87-96, 1948.

[5] A. Bystrom and D. G. Berntsen, "An experimental investigation of cavity-mounted helical antennas," IRE Transactions on Antennas and Propagation, vol. 4, no. 1, pp. 53-58, 1956.

[6] H. E. King and J. L. Wong, "Characteristics of 1 to 8 wavelength uniform helical antennas," IEEE Transactions on Antennas and Propagation, vol. 28, no. 2, pp. 291-296, 1980.

[7] A. R. Djordjevic, A. G. Zajić, M. M. Ilić, and G. L. Stüber, "Optimization of helical antennas [antenna designer's notebook]," IEEE Antennas and Propagation Magazine, vol. 48, no. 6, pp. 107-115, 2006.

[8] A. R. Djordjevic, A. G. Zajić, and M. M. Ilić, "Enhancing the gain of helical antennas by shaping the ground conductor," IEEE Antennas Wireless Propagation Letters, vol. 5, no. 1, pp. 138-140, 2006.

[9] R. J. Stegen, "Impedance matching of helical antennas," IEEE Transactions on Antennas and Propagation, vol. 12, no. 1, pp. 125-126, 1964.

[10] J. D. Kraus, "A 50-ohm input impedance for helical beam antennas," IEEE Transactions on Antennas and Propagation, vol. AP-25, no. 6, pp. 913-913, 1977.

[11] V. Wongpaibool, "Improved axial-mode-helical-antenna impedance matching utilizing triangular copper strip for 2.4-GHz WLAN," in 2008 International Wireless Communications and Mobile Computing Conference, pp. 869-873, Crete Island, Greece, 2008.

[12] M. M. Ilić, S. V. Savić, and P. Djondović, "Internal matching of UHF helical antenna exciters for magnetic resonance imaging," in 3rd International Conference on Electrical, Electronic and Computing Engineering, pp. API1.1.1-API1.1.5, Zlatibor, Serbia, 2016.

[13] D. E. Baker, "Measured performance of a broadband matching section for peripherally fed helical antennas," The Transactions of the South African Institute of Electrical Engineers, vol. 76, no. 2, pp. 56-61, 1985.

[14] R. P. Hecken, "A near-optimum matching section without discontinuities," IEEE Transactions on Microwave Theory and Techniques, vol. 20, no. 11, pp. 734-739, 1972.

[15] B. D. Popović and A. Nešić, "Generalisation of the concept of equivalent radius of thin cylindrical antennas," IEE Proceedings H-Microwaves, Optics and Antennas, vol. 131, no. 3, pp. 153-158, 1984.

[16] A. R. Djordjević, M. B. Baždar, V. V. Petrović, D. I. Olćan, T. K. Sarkar, and R. F. Harrington, Awas for Windows Version 2.0: Analysis of Wire Antennas and Scatterers, Artech House, Norwood, Massachusetts, 2002.

[17] WIPL-D. WIPL-D Pro 11.02013, http://www.wipl-d.com.

[18] B. D. Popović, M. B. Dragović, and A. R. Djordjević, Analysis and Synthesis of Wire Antennas (Antennas Series), John Wiley \& Sons-Research Studies Press, New York, 1982.

[19] A. R. Djordjević, M. B. Baždar, R. F. Harrington, and T. K. Sarkar, Linpar for Windows: Matrix Parameters for Multiconductor Transmission Lines, Version 2.0, Artech House, Norwood, Massachusetts, 1999.

[20] ANSYS, ANSYS MAXWELL 15.0.02014, http://www.ansys. com.

[21] ANSYS, ANSYS HFSS 15.0.02014, http://www.ansys.com.

[22] B. D. Popović, A. R. Djordjević, and N. M. Kirćanski, "Simple method for analysis of dielectric-coated wire antennas," Radio and Electronic Engineer, vol. 51, no. 3, pp. 141-145, 1981. 


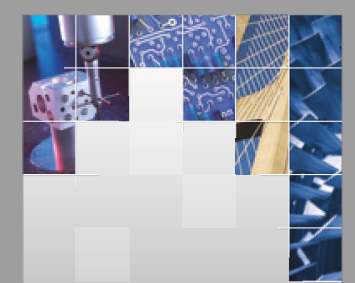

\section{Enfincering}
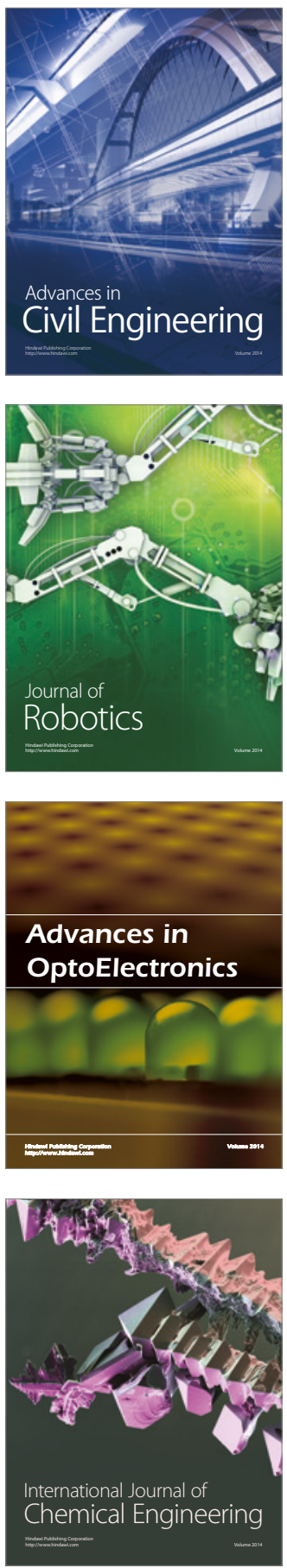

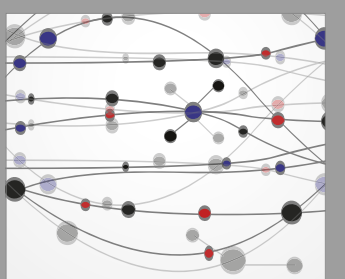

The Scientific World Journal

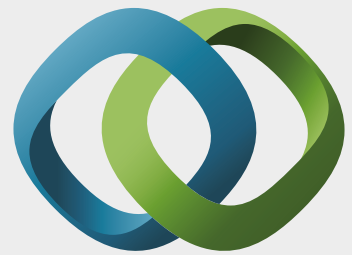

\section{Hindawi}

Submit your manuscripts at

https://www.hindawi.com
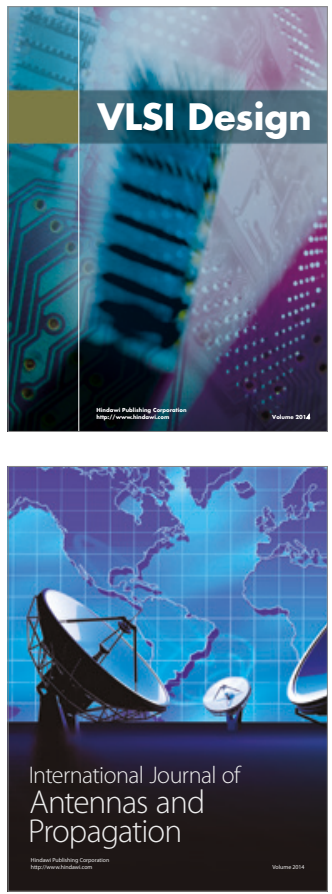

\section{Rotating}

Machinery
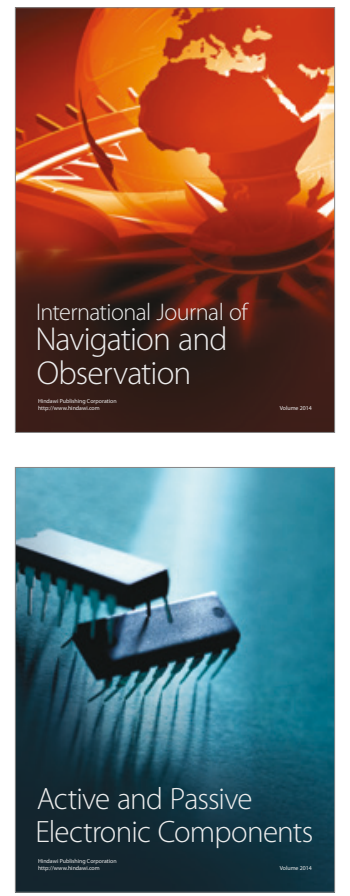
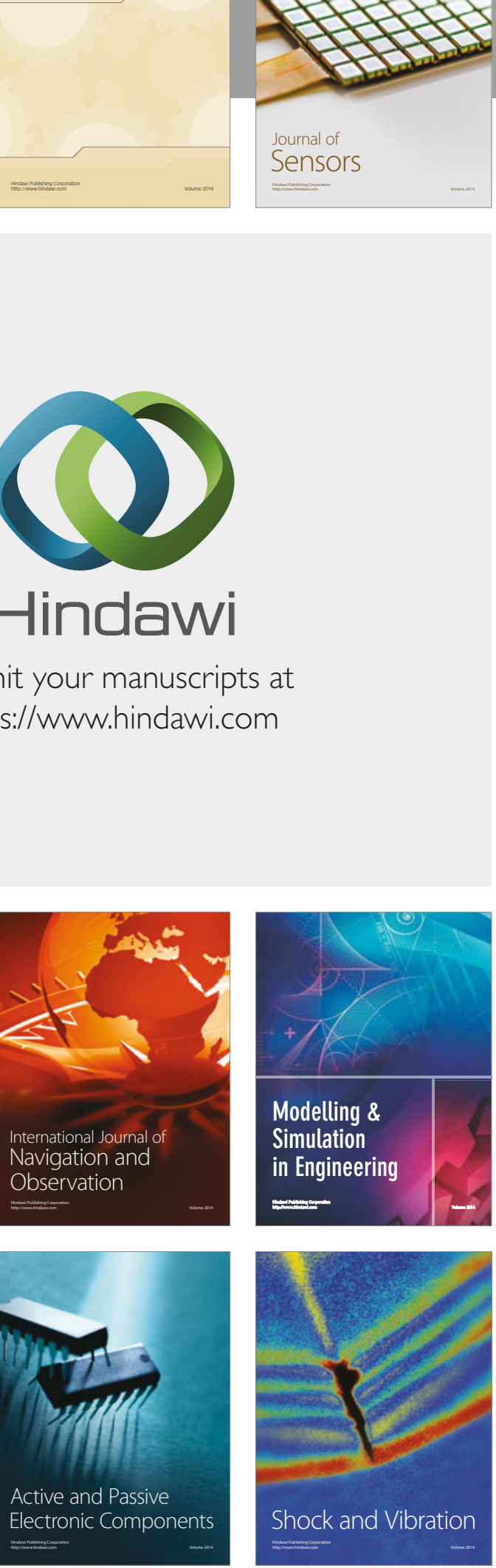
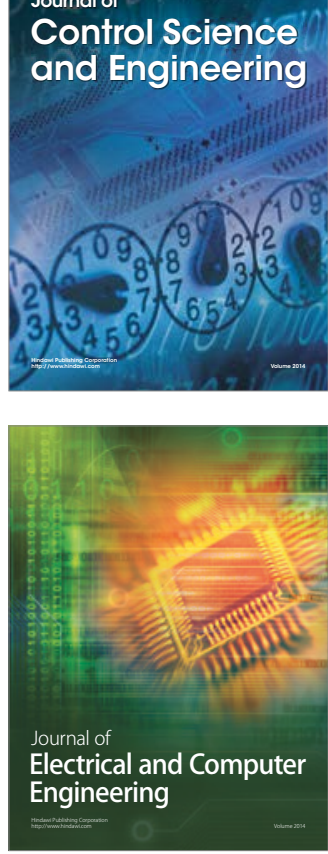

Distributed

Journal of

Control Science

and Engineering
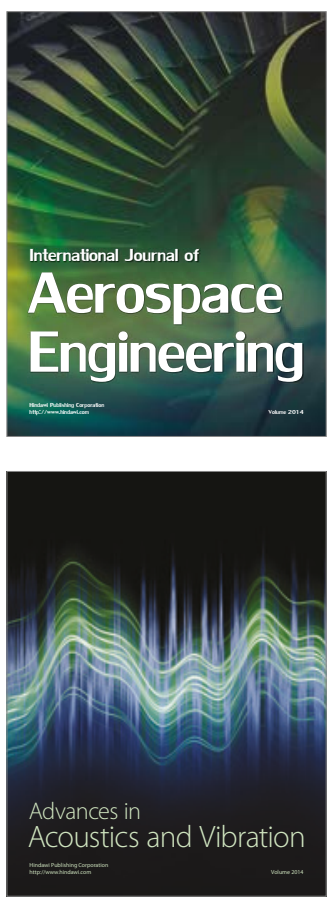

Sensor Networks 